\title{
Hydrogen storage: The major technological barrier to the development of hydrogen fuel cell cars
}

\author{
D.K.Ross \\ Institute for Materials Research \\ University of Salford \\ M5 4WT \\ $U K$
}

\begin{abstract}
In this paper, we review the current technology for the storage of hydrogen on board a fuel cell propelled vehicle. Having outlined the technical specifications necessary to match the performance of hydrocarbon fuel, we first outline the inherent difficulties with gas pressure and liquid hydrogen storage. We then outline the history of transition metal hydride storage, leading to the development of metal hydride batteries. A viable system, however, must involve lighter elements. The first new system to get serious consideration is titanium-activated sodium alanate, followed by the lithium amide and borohydride systems that potentially overcome several of the disadvantages of alanates. Borohydrides can alternatively produce hydrogen by reaction with water in the presence of a catalyst but the product would have to be recycled via a chemical plant. Finally various possible ways of making magnesium hydride decompose and reform more readily are discussed. The alternative to lighter hydrides is the development of physisorption of molecular hydrogen on high surface area materials such as carbons, Metal Oxide Frameworks (MOFs), zeolites etc.. Here the problem is that the surface binding energy is too low to work at anything above liquid nitrogen temperatures. Recent investigations of the interaction mechanism are discussed which show that systems with stronger interactions will inevitably require a surface interaction that increases the molecular hydrogenhydrogen distance.
\end{abstract}

Key words: Hydrogen storage, fuel cells, alanates, lithium amide, sodium borohydride, magnesium hydride, activated carbons, MOFs, zeolites, physisorption of molecular hydrogen.

\section{Introduction}

Both from the point of view of global warming and from that of the inevitable exhaustion of the Earth's oil reserves, it has become highly desirable to develop an alternative energy source for automobiles. Since the development of the PEM (Proton Exchanged Membrane) fuel cell, which is fuelled by hydrogen and oxygen (air) and produces only water, hydrogen has generally been seen to be the most promising approach. However, although the development of fuel cell technology appears to be progressing smoothly towards eventual commercial exploitation, a viable method for storing hydrogen onboard a vehicle is still to be established. The US DoE has taken what is perhaps an extreme view of what the technology needs to deliver. By rejecting taxation as a means of persuading the public to change their fuel, the DoE starts from the position that the 
hydrogen fuelled vehicle must be able to match the performance of a hydrocarbon fuelled car and must do so at a comparable cost (based on the costs of oil in the recent past). This has led them to establish a series of targets which, given the remarkable advantages of petrol and diesel for this purpose, will be very tough to meet [1]

Gravimetric energy density: $2 \mathrm{KWh} / \mathrm{kg}$

Volumetric energy density: $1.5 \mathrm{KWh} / \mathrm{l}$

H2 storage capacity (mass fraction) of $6 \mathrm{wt} \%$ (on a system basis)

Operating temperature: -30 to $+50^{\circ} \mathrm{C}$

Re-fuelling time $<5$ min

Refuelling Rate: $1.5 \mathrm{kgH} 2 / \mathrm{min}$

Recoverable amount of hydrogen: $90 \%$

Cycle life: 500 times (requirements for the physical properties of storage material)

Cost target: \$5/KWh (storage material only, without peripheral components)

From our present point of view, the most significant of these targets are that (1) the fuel tank system when charged should contain $6 \%$ gravimetric hydrogen density; and (2) the system should be capable of being recharged at the filling station in less than 5 minutes. In addition, (3) the hydrogen should be available at the inlet of the fuel cell at a pressure of a few bar without it being necessary to heat the store to a temperature of more than $50^{\circ} \mathrm{C}$. If (2) cannot be met, we could consider using replaceable fuel tanks that can be recharged off-line at the fuel station. If one goes this way, one should also consider the use of chemical hydrides that will liberate hydrogen on the addition of water [1,2] but here the product would have to be sent back to a factory to be recycled.

Four different methods of hydrogen storage are currently being considered, namely liquid hydrogen, high pressure hydrogen, solid state hydride storage (including chemical hydrides) and porous solid adsorption of molecular hydrogen. The first two methods have reached the engineering prototype stage while for the last two methods, there is still much to be done in selecting the optimum system for further development. This paper will concentrate on the present status of these last two approaches. Before doing that, however, we should discuss the disadvantages of the first two methods, given that they have actually been demonstrated to work.

\section{Liquid and High Pressure Hydrogen Storage}

Firstly, liquid hydrogen can be stored on board the vehicle, as has been demonstrated by BMW who have produced a limited series of cars fuelled in this way [3]. In this case, most of the hydrogen is supplied to a conventional Internal Combustion Engine (ICE) with some going to a fuel cell which provides the electrical power for air conditioning etc. This approach yields a gravimetric storage density of $100 \% \mathrm{H}_{2}$. However, if you include the mass of the tank, this is reduced to about $10 \%$ gravimetric $\mathrm{H}_{2}$. Moreover, it has two major disadvantages: (1) there is inevitably a boil-off rate which is currently $1 \% /$ day; (2) there is an inevitable energy loss due to the refrigeration process which amounts to $30 \%$ of the energy available from burning the hydrogen, even in the largest plant size.[4]. 
The second approach is to store the hydrogen at high pressures. A conventional steel hydrogen cylinder achieves about $1 \%$ gravimetric hydrogen. Recent developments of fibre reinforced resin have reached pressures of $700 \mathrm{bar}$, corresponding to about half the density of liquid hydrogen. The CUTE hydrogen fuelled buses [5], as have been operating recently in London, actually use conventional steel hydrogen cylinders (150 bars). However, while we are all happy to have buses driving round with high pressure hydrogen cylinders on board - after all, gas suppliers are doing this all the time using properly designed lorries - there are some anxieties about letting the general public loose with the really high pressure cylinders that are now being developed.

\section{Metal Hydride Stores}

Turning to metal hydride stores, here the hydrogen split into atoms at the surface of the metal and then enters the metallic lattice in the atomic form, diffuses through the metal, jumping between interstitial sites; and finally forms a hydride phase with a more or less ordered hydrogen sub-lattice. If the (inter) metallic lattice contains $d$ or $f$ electron states at the Fermi surface, the hydrogen s electron can be partly donated to the conduction band and the bare proton, screened electrostatically by electrons at the Fermi Surface, can move relatively freely through the lattice. Most binary or intermetallic hydrides are either very stable or very unstable but a number do show an equilibrium hydrogen pressure at room temperature of about 1 bar. The prime example of such a system (apart from palladium, which is rather too expensive!) is lanthanum nickel hydride $\left(\mathrm{LaNi}_{5} \mathrm{H}_{6}\right)$. This system was first investigated by Buschow and Miedema at Philips in Eindhoven [6] and subsequently, its use in rechargeable metal hydride batteries operated in $\mathrm{KOH}$ was demonstrated by Notten [7] $. \mathrm{LaNi}_{5} / \mathrm{H}$ is not itself a practical system for a battery as the lattice tends to fragment after a few hydrogenation cycles but, in the following years, a series of $\mathrm{AB}_{5}$ compounds based on this system were developed, mainly in Japan [8]. Batteries made from these compounds (called Nickel - Metal-hydride (NiMH) batteries).have largely replaced Ni-Cd rechargeable batteries. Indeed, in China, which has large deposits of rare earths, bicycles with electric motors powered by metal hydride batteries are in mass production [9]. However, batteries on their own have to be recharged by plugging into the mains electricity over night- and $\mathrm{AB}_{5}$ compounds can only provide about $1 \%$ hydrogen by mass - so, while they have a role for powering bicycles, they do not seem capable of providing a long term substitute for petrol or diesel fuel.

The hunt is therefore on to find lighter metal lattices that will work as well as these AB5 materials while satisfying the gravimetric storage requirement. However, there are a number of major problems that will have to be dealt with. Firstly, the protonic transport system that works for the transition metal hydrides (where the metal atom effectively changes valency as the hydrogen is added) is not available where the bonding is either ionic $\left(\mathrm{H}^{-}\right)$or covalent - because here the chemical reaction involves the transfer of intregal numbers of electrons. Hence the addition of hydrogen must involve the complete geometric rearrangement of the lattice. This inevitably results in a much smaller diffusion coefficients for hydrogen in the solid phases and hence a practical system has to involve nanoscale materials with large surface areas. Secondly, most potential systems are rather too thermodynamically stable. This fact has two consequences; firstly the 
hydrides have to be heated up to an inconveniently high temperature to provide hydrogen at an appropriate pressure and, secondly, the heat of absorption (while recharging with hydrogen) is rather high and this means that a large amount of heat has to be removed during the refuelling process, making it difficult to meet the fuelling time specified by the DoE (5 minutes). Simple calculations show that if the refuelling is to take place in this time, a typical American car would require several megawatts of cooling during the refuelling process. A third difficulty is that the light hydride systems considered so far are all rather strong reducing agents and hence react vigorously with air. Apart from the possibility of dust explosions, this means that the fuel will lose its activity if there is any inward leakage of air. Vacuum-tight engineering will therefore be essential. Here again, the possibility of a system that involves changing tanks at the filling station, as, for example, with Calor Gas, may have to be considered.

\subsection{Sodium alanate system}

The first light hydride system to show promise was sodium alanate $\left(\mathrm{NaAlH}_{4}\right.$ and $\mathrm{Na}_{3} \mathrm{AlH}_{6}$ ). Bogdanovich and Schwickardi showed that when these systems are activated using $\mathrm{TiCl}_{3}$, the rate of absorption/desorption of hydrogen is much enhanced [10]. The full reaction can be written:

$$
3 \mathrm{NaAlH}_{4} \leftrightarrow \mathrm{Na}_{3} \mathrm{AlH}_{6}+2 \mathrm{Al}+3 \mathrm{H}_{2} \leftrightarrow 3 \mathrm{NaH}+3 \mathrm{Al}+9 / 2 \mathrm{H}_{2}
$$

The hydrogen is evolved over two plateaus. The readsorption process also shows two plateaus (3.7\% in the first plateau and 3\% in the second) but there is considerable hysteresis in this process. The performance can be improved in a variety of ways, for instance by mechanically alloying the material [11].

This system illustrates nicely the difference between the light hydrides and the traditional transition metal systems in that the hydrogen is evolved as a result of a complete change in the structure in which a single phase $\left(\mathrm{NaAlH}_{4}\right)$ decomposes into the hexahydride and aluminium metal where the two alanate phases have quite different crystal structures. It is quite remarkable that in this process, the $\mathrm{Al}$ released collects in precipitates but it is even more remarkable that in the reverse reaction, when hydrogen pressure is applied, the $\mathrm{Al}$ precipitates decompose to reform the $\mathrm{NaAlH}_{4}$ phase. It seems likely that some volatile species is formed by direct reaction of hydrogen with the small Al precipitates and that this allows the reaction to proceed at a reasonable rate. It is also quite difficult to understand the precise role of the $\mathrm{Ti}$ - whether it is a surface catalyst or whether it is incorporated in the crystal structure [12].

\subsection{The Lithium Imide System}

A very similar process has been demonstrated in the Lithium imide system, starting from a mixture of lithium amide and lithium hydride [13].

$$
\mathrm{LiNH}_{2}+2 \mathrm{LiH} \leftrightarrow \mathrm{Li}_{2} \mathrm{NH}+\mathrm{H}_{2}+\mathrm{LiH} \leftrightarrow \mathrm{Li}_{3} \mathrm{~N}+2 \mathrm{H}_{2}
$$

The lithium amide and the lithium hydride can be ball-milled together to ensure that the lithium hydride is close enough to react rapidly with the amide. Alternatively, we can 
start with lithium nitride and hydrogenate it. Doing it this way automatically produces an intimate mixture of amide and hydride. Here again, there is a volatile molecule that facilitates the reaction. In this case, it is $\mathrm{NH}_{3}$.

The disadvantage of the LiN system is that the amide and imide are rather too stable. For instance, the thermal desorption profiles under argon show a first peak at $700 \mathrm{~K}$. It is therefore necessary to find ways of destablising this structure, for instance by adding $\mathrm{Mg}$, which reduces the temperature range for thermal desorption in proportion to the amount of $\mathrm{Mg}$ added up to the point where $30 \%$ of the lithium has been replaced by magnesium. Here the desorption temperature has been reduced to 350K[14].

\subsection{Borohydride}

A similar system with some potential for development is the borohydride system which shows the highest known hydrogen contents. In the case of lithium borohydride [15], the hydrogen content is $18.5 \%$ by mass but the reversible hydrogen content is actually $13.8 \%$ as the decomposition is given by the equation

$\mathrm{LiBH}_{4} \leftrightarrow \mathrm{LiH}+\mathrm{B}+(3 / 2) \mathrm{H}_{2}$

and lithium hydride is far too stable relative to Li metal to decompose at accessible temperatures. The decomposition above takes place around 573K, which is actually above the melting point of $\mathrm{LiBH}_{4}$ which is $550 \mathrm{~K}$. However, the decomposition process is enhanced by use of a catalyst. For instance, $\mathrm{SiO}_{2}$ has been reported to reduce the decomposition temperature to 473K [16]. It has also been shown that the rehydriding process will proceed to completion under $35 \mathrm{MPa}$ at $873 \mathrm{~K}$ [17]. It is interesting to note that XRD measurements after the decomposition only show small peaks due to $\mathrm{LiH}$. Presumably the boron remains in an amorphous form. Equally, after rehydriding, the peaks corresponding to $\mathrm{LiBH}_{4}$ are small and rather broad. However, the authors demonstrated that the $\mathrm{BrH}_{4}{ }^{-}$anions are in fact being formed because the characteristic vibrational modes of $\mathrm{BH}_{4}{ }^{-}$are seen in the Raman spectra both before dehydriding and after rehydriding. Here again, current efforts are being devoted to surveying different mixtures of alkali cations to see if the compound can be sufficiently destablised to release and reabsorb hydrogen at lower temperatures and useable pressures. However, given that the hydrogen is part of a covalently-bonded anion, it is not obvious that changing the cation will have a very significant effect on the stability.

An alternative way of using sodium borohydride is to make use of its reaction with water in an alkaline solution in the presence of a catalyst, for instance ruthenium. This process has been developed, for instance, by Millenium Cell Inc [18]. Here the reaction is

$$
\mathrm{NaBH}_{4}+2 \mathrm{H}_{2} \mathrm{O} \rightarrow 4 \mathrm{H}_{2}+\mathrm{NaBO}_{2}+300 \mathrm{~kJ}
$$

If the solution starts at the maximum concentration, the gravimetric yield of hydrogen is about $7.5 \%$. Because the catalyst is heterogeneous, the solution can be added to start the reaction and then, if necessary removed to stop it. In practice, the product, sodium metaborate solution, would be drained away from the catalyst at the filling station and 
would be replaced by fresh sodium borohydride solution. The hydrogen produced by this route is ideal for use with a PEM fuel cell being very pure, except for water vapour which is actually necessary to humidify the PEM membrane.

This is a very attractive route for storing hydrogen on board a vehicle. The solution of sodium borohydride is quite inert - clearly safer than gasoline. The main problem is the cost of the borohydride which is quoted to be $\$ 80 / \mathrm{kg}$. Thus the main research activity in this area is in the development of alternative processes for regenerating $\mathrm{NaBH} 4$ from the $\mathrm{NaBO} 2$, presumably at some central plant. However, it must be realized that the heat released, $75 \mathrm{~kJ} / \mathrm{mole} \mathrm{H} 2$ is not used directly for propulsion so that the efficiency of the process is inevitably somewhat limited in energy terms. Thus, while being very attractive for small portable devices, this process still seems somewhat impractical for vehicle propulsion systems.

\subsection{Magnesium hydrides}

Magnesium hydride is the most studied of the light hydrides as it is potentially cheap. The stoichiometric material suffers from both rather high stability and very slow hydrogen absorption/evolution due to low hydrogen diffusion constants in the bulk. The obvious remedy to low diffusion rates is to reduce the particle size by ball-milling (the evolution time being approximately $\mathrm{l}^{2} / \mathrm{D}$, where $\mathrm{l}$ is the particle dimension and $\mathrm{D}$ is the chemical diffusion coefficient). Indeed, 15 minutes of ball milling of the as- received material reduces the evolution time to about $2000 \mathrm{sec}$ at $575 \mathrm{~K}$ and $0.1 \mathrm{kPa}$ [19]. However, a very much more dramatic reduction in the evolution time can be achieved by adding about $10 \%$ by weight of nanoscale $\mathrm{Nb}_{2} \mathrm{O}_{5}$. Here, after 15 minutes of ball milling, the evolution time is reduced to about $100 \mathrm{sec}$, a practical value. Re-absorption took place at $1 \mathrm{MPa}$ at $300^{\circ} \mathrm{C}$. This suggests that $\mathrm{MgH}_{2}$ is rather too stable and that this is not significantly affected by the addition of the $\mathrm{Nb}_{2} \mathrm{O}_{5}$. However, it is possible that a lower stability compound can be produced by starting from a magnesium alloy or intermetallic compound.

\section{Physisorbed hydrogen on high surface area materials}

The other possible method of storage is to physisorb molecular hydrogen on a solid with a high surface area - as in a typical molecular sieve. This approach works well with methane which can be efficiently stored on activated carbons. However, for hydrogen and for heats of adsorption that are typical of Van der Waals interactions, useful storage at ambient temperatures looks unlikely. In fact, simple calculations show that, at ambient temperatures, the amount stored in a unit volume increases with hydrogen pressure but, at practical pressures, rather less would be stored than would be stored in the same volume of gas at the same gas pressure. On the other hand, if the hydrogen molecule splits into two and each resulting hydrogen atom is chemisorbed, the strength of the chemical bond is normally such that it is impossible to get the hydrogen to desorb at reasonable temperatures and pressures.

There are two possible ways round this difficulty. The first would be to store the hydrogen in an adsorber at $80 \mathrm{~K}$. The second is to find a surface where the isosteric heat 
of adsorption is significantly greater than for the normal physisorption case (about 4 $\mathrm{kcals} / \mathrm{mole}$ in chemist's units or $40 \mathrm{meV} /$ atom for physicists). Some years ago, a great deal of excitement was generated by reports that very large amounts of hydrogen could be stored on carbon nanotubes [20] and carbon nanofibres [21] at room temperature, suggesting a dramatic increase in the heat of adsorption but these results have not proved to be reproducible [22].

Consider first the use of a store kept at nitrogen temperatures. The amount of hydrogen stored on a surface can be measured using either a gravimetric or volumetric technique. Comparative gravimetric and volumetric data show comparable isotherms [23]. These have a typical Langmuir shape, suggesting that the asymptotic mass adsorbed corresponds to a monolayer coverage. It is not surprising that multi-layer coverage is not clearly seen so far above the boiling point of liquid hydrogen. Obviously, in measuring the uptake of hydrogen at $80 \mathrm{~K}$, one has to be careful that no other gas is present. Some of the early spurious results were probably due to irreversibly trapping water vapour. It is clearly essential to first bake out the sample at high temperature and then to confirm that the measured isotherm is reversible. An interesting point to note about the gravimetric technique is that, before correction for the buoyancy effect (due to different masses of gas being displaced by the sample and the counter balance), the mass adsorbed first increases along a typical Langmuir-type isotherm but then begins to lose mass linearly due to the uplift of the gas around the sample. If the buoyancy correction is made correctly, the corrected curve will tend asymptotically to a maximum amount of gas adsorbed as expected for a Langmuir isotherm. However, the useful effect of the adsorber (the improvement in storage compared to a pressurised gas in an empty volume) is given by the actual increase in the weight of the sample without buoyancy correction.

As would be expected for a limiting monolayer coverage, the mass of hydrogen adsorbed at $80 \mathrm{~K}$ turns out to be proportional to the surface area as measured using the BET (Brunauer, Emmet and Teller) method, more or less regardless of the nature of the surface, being about $2.5 \%$ per $1000 \mathrm{~m}^{2}$ surface area[23]. However, at ambient temperatures and 20 bars pressure, only about $7 \%$ of the $80 \mathrm{~K}$ mass is adsorbed. While manufacturers would be reluctant to operate a fuel tank at $80 \mathrm{~K}$, such a system would have significant advantages over a liquid hydrogen store at $20 \mathrm{~K}$ in that the refrigeration costs would be about $25 \%$ of those at $20 \mathrm{~K}$ and the boil-off would be slower and could be accommodated by allowing for a slow increase in overall hydrogen pressure. Further, because the heat of adsorption is small, heat release during fuelling is not a problem. Also, in accident conditions, the rate of release from a porous store would be much slower than from a liquid hydrogen or gas pressure storage system.

In order to have any serious chance of a viable molecular hydrogen storage system, one would need to develop a surface that has a higher heat of adsorption than a typical carbon surface and hence we need to understand the physics of the surface interaction on a welldefined surface such as carbon nanotubes. The physics of this process can be investigated in a very direct way using Inelastic Neutron Scattering [24, 25]. The beauty of this method is that the inelastic scattering is dominated by the para-ortho inelastic neutron scattering cross section. If the SWNT (Single-Walled Nano Tube) sample is held at 20K 
or so, all the hydrogen converts into the para- state with zero angular momentum $(l=0)$. Using the high resolution TOSCA spectrometer at ISIS at the Rutherford Appleton Laboratory - which measures neutron energy loss processes down to a final energy of 3.5 $\mathrm{meV}$, we can measure the neutron energy loss cross section associated with exciting paramolecules $(\mathrm{l}=0)$ to ortho- $(\mathrm{l}=1)$ states, which for free molecules, would involve a neutron energy loss of $14.7 \mathrm{meV}$. However, the measurements (Fig 1) show a complex peak shape, suggesting one or more non-spherical potential energy wells at the trapping site. By measuring this spectrum as a function of hydrogen coverage, it was concluded that there were at least two different surfaced sites. The first to be filled, attributed to a groove site where two surface nanotubes touch, corresponds to a split peak (13.5 and $15.1 \mathrm{meV}$ with a 2:1 intensity ratio). The second, at $14.5 \mathrm{meV}$ (unsplit) is attributed to sites on the external convex surface of the nanotubes. As the surface coverage exceeds $100 \%$, a third peak appears at about $14.6 \mathrm{meV}$ which is attributed to a second layer of molecules. By comparing the temperature dependence of the relative intensity of the groove site peaks with the other two features as a function of hydrogen coverage, it is concluded that the former have an isosteric heat of $\mathrm{H}_{2}$ adsorption of $75 \mathrm{meV}$ compared with $35 \mathrm{meV}$ for the external convex surface. This compares with $39 \mathrm{meV}$ on the equivalent site on a graphene surface. It is interesting to note that the reduced energy peaks of the groove site imply a slight increase in the $\mathrm{H}-\mathrm{H}$ distance and corresponding increase in the moment of inertia. This corresponds to a slight reduction in the zero point energy of the rotational states which presumably contributes to the increased heat of adsorption on these sites. This immediately suggests that surfaces can be produced that increase the strength of the interaction so as to increase the $\mathrm{H}-\mathrm{H}$ distance by a much greater amount. Such an interaction might also give rise to a reduction in the zero point energy for the $\mathrm{H}-\mathrm{H}$ vibration frequency which could give rise to a significant increase in the trapping energy.

The search for a viable high surface area storage material has of course extended from carbon systems to, for instance, zeolites, Metal Oxide Frameworks (MOFs) and other materials developed for use as catalyst supports, supercapacitors etc. .

\section{Conclusions}

The search for a material that is capable of storing hydrogen in the amounts necessary to make a hydrogen-fuelled fuel cell car a practical proposition has become a major objective of materials research. The DoE targets of $6 \%$ by mass, combined with adequate rates of refuelling the vehicle, will clearly be very tough to meet. Whether the solution is found amongst the light hydrides or through a porous store kept at $80 \mathrm{~K}$, the challenges for maintaining the storage material in an active condition will pose a considerable in the engineering of a satisfactorily vacuum-tight containment.

\section{References}

[1] FreedomCAR and Fuel Technical Partnership: Technical goals. www.eere.energy.gov/vehiclesandfuels/about/partnerships/freedomcar/index.shtml [2] A.W. McLaine, ER.W.Breault, C. Larsen, R.Konduri, J.Rolfe, F.Becker and G.Miskolery; Proc 2000 US DoE Hydrogen Program Review, NREL/CP-570-28890 
[3] http://www.bmwworld.com/models/750hl.htm

[4] U. Bossel, B. Eliason and G.Taylor,

http://www.evworld.com/library/h2economyFinalReport.pdf

[5] Clean Urban Transport for Europe

http://www.fuelcelltoday.com/FuelCellToday/IndustryDirectory/IndustryDirectoryExtern

al/IndustryDirectoryDisplayCompany/0,1664,1064,00.html

[6] Buschow K. H. J. and A. R. Miedema: in Hydrides for Energy Storage, A. F.

Andresen and A. J. Maeland , eds., Oxford:Pergamon .1978; 235:

[7] Notten PHL. J. Electrochemical Soc.1991 $138: 1877$

[8] Sakai T, Uehara, I, . Ishikawa H. J. Alloys Comp 1999; 293-295: 762.

[9] e.g Hangzhou Lanbei Electric Bicycle Co. Ltd.

http://lanbei.en.alibaba.com/product/50091127/50535161/Electric_bicycle/Electric_Bicy

cle_Standard_Pedal_Two_Groups_of_Battery_html

[10] Bogdanovic B and Schwickardi, M. J. Alloys Comp 1997; 253-254:1; Bogdanovich

B, Brand RA, Marjanovic A, Schwickardi M Tolle J. J. Alloys and Comp 2000; 302: 36.

[11] Kircher, O and Fichtner MJ. Alloys and Comp 2005; 404-406: 339.

[12] Andrei CM, Walmsley J, Brinks H.W, Homestad R, Jensen CM, Hauback BC. Appl. Phys. A. 2005; 80: 709.

[13] Chen P, Xiong ZT, Luo JZ, Lin JY, Tan KL. Nature 2002; 420: 302.

[14] Nakamori Y, Kitahara G, Miwa K, Ohba N, Noritake T, Towota S, Orimo S. J.

Alloy and Comp2005; 404-406:396

[15] Zuettel A, Wenger P, Rentsch S, Sudan P, Mauron Ph, Emmenegger Ch. J Power

Sources 2003; 5192: 1

[16] Zuettel A, Rentsch P, Fisher P, Wenger P, Sudan P, Mauron Ph, Emmenegger Ch. J. Alloys Comp 2003; 356/357: 515

[17] Orimo S, Nakamori Y, Kitahara G, Miwa K, Ohba N, Towata S, Zuettel A. J.

Alloys and Comp 2005;404-406:427

[18] Amendola SC, Sharp-Goldman SL, Janjua MS, Spencer, NC, Kelly MT, Petillo PJ, Binder M, Int J Hydrogen Energy 2000;25:969

[19] Freidrichs O, Klassen T, Sanchez-Lopez JC, Bormann R, Fernandez A. Scripta Materia 2006; 54:1293

[20] Dillon AC, Jones KM, Bekkedalh TA, Klang CHBethune DS, Heben MJ. Nature 1997; 386:377

[21] Chambers A, Park C, Baker RTK, Rodriguez NM. J Phys Chem B 1998; 102:4253

[22] Schimmel HG, Nijkamp G, Kearley GJ, Rivera A, de Jong KP, Mulder FM. Mat Sci.

Eng. B 2004; 108:124

[23] Anson A, Benham, M, Jagiello J, Callejas,MA, Benito AM, Maser, WK, Zuttel, A, Sudan P, Martinez MT. Nanotechnology 2004; 15:1503

[24] Georgiev PA, Ross DK, De Monte A, Montaretto-Marullo U, Edwards RAH, Ramires-Cuesta AJ, Colognesi D. J Phys. Condens Matter 2004; 16: L 73-78

[25] Georgiev PA, Ross DK, De Monte A, Montaretto-Marullo U, Edwards RAH, Ramirez-Cuesta AJ, Adams MA Colognesi D. Carbon 2005 ; 43 : 895 
Figures 


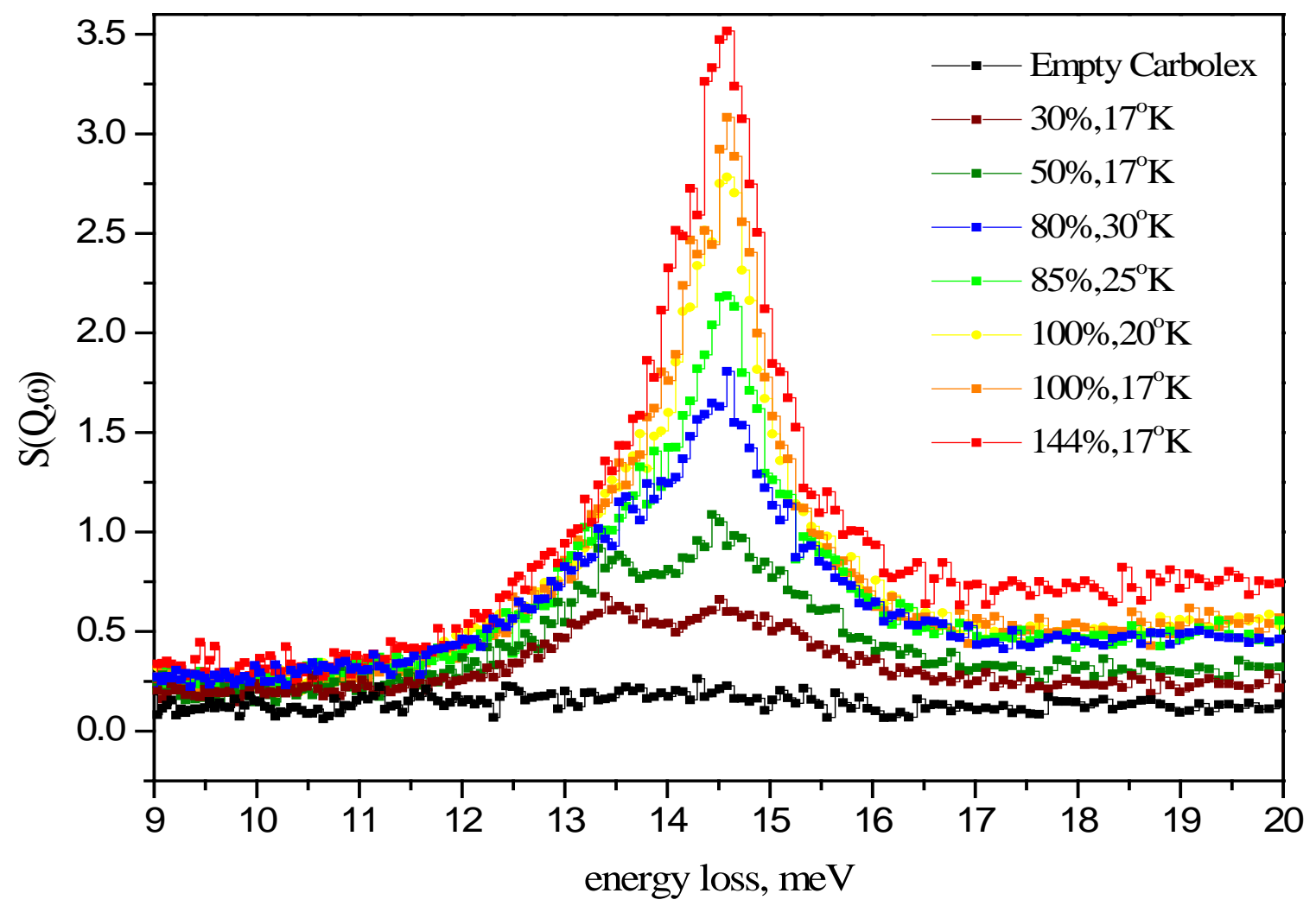

Fig 1. Inelastic neutron scattering data measured from hydrogen molecules adsorbed onto a sample of single walled carbon nanotubes (Carbolex Selected Grade) in the vicinity of the $\mathrm{l}=0$ to $\mathrm{l}=1$ rotational level for a series of different surface coverages [24] . The vertical scale is $\mathrm{S}(\mathrm{Q}, \omega)$, the scattering intensity as a function of momentum transfer ( $\hbar \mathrm{Q})$ and energy transfer $(\hbar \omega)$. For the lowest coverage, the molecules are mainly trapped on the groove sites which give the split peak. For higher coverages, the additional molecules are trapped at normal sites on the convex surface of the nanotubes. Because the potential on the latter sites is not perturbed, the energy diference is close to the $14.7 \mathrm{meV}$ value expected fro a free molecule. 
